\title{
Demethylation of miR-495 inhibits cell proliferation, migration and promotes apoptosis by targeting STAT-3 in breast cancer
}

\author{
YI CHEN, DONGLIN LUO, WUGUO TIAN, ZHIRONG LI and XIAOHUA ZHANG \\ Department of General (Breast and Thyroid), Daping Hospital of the Third Military Medical University, \\ No. 10 Yangtze River Branch, Yuzhong, Chongqing 400042, P.R. China
}

Received November 2, 2016; Accepted April 18, 2017

DOI: $10.3892 /$ or.2017.5621

\begin{abstract}
In breast cancer (BC), silencing of miRNA genes due to miRNA gene promoter methylation are the important mechanisms directly contributing to tumorigenesis and tumor progression. miRNA-495 (miR-495) has been reported to be a tumor suppressor gene in various cancers, but its role and regulation in $\mathrm{BC}$ remains unclear. In the present study, the level of miR-495 was inversely correlated with the expression of STAT-3 in BC tissues and cell lines. miR-495 can directly target 3'-UTR of STAT-3 mRNA and thereby decrease the expression of STAT-3 in MCF-7 and HCC1973 cells by Targetscan and Dual-luciferase assay. We further analyzed miR-495 promoter methylation by sodium bisulfite sequencing method (BSP), and found DNA methyltransferase inhibitor, 5-AzaC concomitantly upregulated expression of miR-495 and downregulated its target gene STAT-3 and its downstream target VEGF. Furthermore, we further observed that 5-AzaC treatment, miR-495 mimics and STAT-3 knockdown significantly inhibited cell function in breast cancer by Transwell assay, EdU flow cytometry, Annexin V-FITC/PI combined with flow cytometry and Hoechst staining. Taken together, our data are first to demonstrate that the miR-495 is silenced due to promoter methylation in breast cancer. DNA methyltransferase inhibitor 5-AzaC could reverse miR-495 (suppressor gene) and STAT-3 (oncogene). The anticancer properties of 5-AzaC were preliminarily confirmed in breast cancer.
\end{abstract}

\section{Introduction}

Breast cancer (BC), is the first ranked morbidity and mortality among female cancer in most countries worldwide (1) and is still rapidly increasing in developing Asian countries (2). The early diagnostic method and synthetical treatment (targeted

Correspondence to: Dr Xiaohua Zhang, Department of General (Breast and Thyroid), Daping Hospital of the Third Military Medical University, No. 10 Yangtze River Branch, Yuzhong, Chongqing 400042, P.R. China

E-mail: 29780293@qq.com

Key words: breast cancer, miR-495, methylation, STAT-3, 5-AzaC therapies combine with chemoradiotherapy) of $\mathrm{BC}$ has progressed enormously, however, more than half of cancer cells will either rapidly acquire multidrug resistance after chemoradiotherapy or are intrinsically multidrug resistant (3). Anomaly epigenetic modification, including methylation of genomic or mitochondrial DNA, and acetylation or methylation of histone significantly alter gene expression, as well as microRNA (miRNA) and is associated with carcinogenesis and multi-drug resistance (MDR) of the cancer cells (4-7). In $\mathrm{BC}$, miRNAs gene silencing due to miRNA gene promoter methylation is the important mechanism directly contributing to chemoresistance (8-13). For example, due to miR-27b promoter methylation, it is significantly downregulated in tamoxifen-resistant cells (MCF-7/TamR) compare with tamoxifen sensitive cells (MCF-7/TamS) and enhances tamoxifen sensitivity by suppression of HMGB3 (10). In addition, $\mathrm{Xu}$ et al (14) reported that super-methylation of miR-152 and miR-148a promoter is present in BC cell lines and tumor tissues compared with the immortalized breast epithelial cells and normal duct and result in BC development through re-expression of IGF-IR and IRS1.

Actually, miR-495 has previously been identified as an important miRNA modulating tumor cell function. Most research has reported that miR-495 usually acts as a tumor suppressor gene in numerous cancer cells (15-23). For instance, Li et al (24) reported that hypermethylationmediated suppression of miR-495 promotes the motility of SGC7901 and MKN28 cells by re-expressing phosphatase of regenerating liver-3. A few studies proved that abnormal expression of miR-495 is inversely associated with multidrug resistance in non-small cell lung cancer (NSCLC) and chronic myelogenous leukemia (CML) by targeting MDR1 or ATP7A $(25,26)$. However, it is unclear whether methylation of miR-495 promoter was involved in the regulation of miR-495, and STAT-3 was regulated by miR-495 in the human BC cells.

In this regard, the aims of the present study were: i) to determine whether the abnormalities of miR-495 (promoter hypermethylation and expression) and its target gene STAT-3 were associated with the development of BC cells by specific target gene; and ii) to determine whether DNA methyltransferase inhibitor 5-AzaC could inhibit BC cell growth by re-expression of miR-495. In this study, we demonstrated that the anticancer properties of 5-AzaC in BC cells in vitro 
may be considered a topic for further experimental studies based on the possibility it could be a novel strategy for cancer therapy in $\mathrm{BC}$.

\section{Materials and methods}

$B C$ cells culture and BC tissues. The human $\mathrm{BC}$ cell lines MCF-7 (cat. no. TCHu 74), HCC1973 (cat. no. TCHu148), the immortalized breast epithelial MCF10A (cat. no. SCSP-575), and human embryonic kidney 293T (cat. no. SCSP-502) were purchased from the Cell Bank of Chinese Academy of Sciences (Shanghai, China). The BC cells were cultured in RPMI-1640 medium (Gibco, Carlsbad, CA, USA), containing $10 \%$ fetal bovine serum (FBS; BI, cat. no. 04-001-1 A) and 293 T cells were maintained in 10\% FBS Dulbecco's modified Eagle's medium (DMEM; Gibco). All cells were cultured in a humidified atmosphere of $5 \% \mathrm{CO}_{2}$ at $37^{\circ} \mathrm{C}$. The human $\mathrm{BC}$ tissues and adjacent tissues were derived from Daping Hospital of the Third Military Medical University. None of the patients received any therapy before the operation. The tissular samples were washed with ice-cold normal saline and cryopreserved in liquid nitrogen immediately. All tissular samples were taken with informed consent and were ratified by the Ethics Committee of the hospital.

Real-time quantitative polymerase chain reaction (RT-qPCR). Total RNA were isolated from BC tissue or cellular samples using TRIzol reagent (Invitrogen, Carlsbad, CA, USA) and converted into cDNA by using Bestar ${ }^{\mathrm{TM}}$ qPCR RT kit (DBI Bioscience, Ludwigshafen, Germany) following the manufacturer's protocol with some modification. The specific RT primers used were: miR-495, 5'-CTCAACTGGTGTCGTGG AGTCGGCAATTCAGTTGAGCGAAAAT-3'; U6snRNA, STAT-3 or GAPDH, primer mix (oligo $\mathrm{dT}_{18}$ and random primer $\mathrm{N}_{6}$ ). The qPCR primers were as follows: GAPDH, forward, 5'-TGTTCGTCATGGGTGTGAAC-3' and reverse, 5'-ATGG CATGGACTGTGGTCAT-3'; STAT-3, forward, 5'-AGAGAT GTGGGAATGGGGG-3' and reverse, 5'-CAACTGGCAAG GAGTGGGTC-3'; miR-495, forward, 5'-ACACTCCAGCT GGGGAAGTTGCCCATGTT-3' and reverse, 5'-CTCAACT GGTGTCGTGGA-3'; U6 snRNA, forward, 5'-CTCGCTTCG GCAGCACA-3' and reverse, 5'-AACGCTTCACGAATTT GCGT-3'. Real-time quantitative PCR was performed using SYBR-Green PCR Master Mix (Bio-Rad Laboratories, Inc., Hercules, CA, USA) to measure relative STAT-3 and miR-495 levels. The PCR condition were as follows: initial denaturation, $94^{\circ} \mathrm{C}$ for $5 \mathrm{~min}$ and then 40 cycles of denaturation, $94^{\circ} \mathrm{C}$ for $30 \mathrm{sec}$, annealing, at $60^{\circ} \mathrm{C}$ for $30 \mathrm{sec}$ and extension, at $72^{\circ} \mathrm{C}$ for $30 \mathrm{sec}$. GAPDH and U6 snRNA was used as an endogenous control for STAT-3 and miR-495 respectively. The data were analyzed using $2^{-\Delta \Delta \mathrm{Ct}}$ method.

Western blot analysis. Total protein was extracted from BC tissue or cellular samples using RIPA lysis (Beyotime Institute of Biotechnology, Beijing, China). The protein concentration was measured by the BCA kit according to the manufacturer's protocol. The equal quality of cell lysate were separated by a $10 \%$ sodium dodecyl sulfate polyacrylamide gel electrophoresis (SDS-PAGE), and then transferred onto polyvinyl fluoride (PVDF) membranes (Bio-Rad Laboratories). The membranes were blocked with 5\% skim milk in TBST (Tris-buffered saline containing $0.1 \%$ Tween-20) at room temperature for $1 \mathrm{~h}$ and incubated with anti-STAT-3, anti-VEGF and GAPDH rabbit antibody (Cell Signaling Technology, Danvers, MA, USA) at $4^{\circ} \mathrm{C}$ overnight. Then, the membranes were washed with TBST three times $/ 5 \mathrm{~min}$, and incubated with horseradish peroxidase (HRP)-conjugated goat anti-rabbit antibody (Santa Cruz Biotechnology, Santa Cruz, CA, USA) at room temperature for $1 \mathrm{~h}$. After being washed, signal intensity was measured by the ECL-PLUS/kit (cat. no. RPN2132; Amersham Biosciences, Little Chalfont, UK) following the manufacturer's protocol. The optical density of bonds was quantified by Quantity One software (Bio-Rad, Laboratories).

Cell transfection. miR-495 mimics and scramble miRNA (NC) were synthesized by Shanghai GenePharma, Co., Ltd. (Shanghai, China). Scramble siRNA (NC) and siRNA for STAT-3 (si-STAT-3), obtained from Santa Cruz Biotechnology miRNA or siRNA were transfected into BC cells using Lipofectamine 2000 (Invitrogen). Specifically, $50 \mathrm{nM}$ of miR-495 mimics/NC or si-STAT-3/NC and $2 \mu 1$ Lipofectamine 2000 were mixed in $200 \mu \mathrm{l}$ Opti-MEM (Invitrogen) and standing $15 \mathrm{~min}$ at room temperature, and then added into the logarithmic phase of cells in a 6-well plate. Cells were washed twice with pre-cooled phosphate-buffered saline (PBS) and harvested at $48 \mathrm{~h}$ post-transfection.

Plasmid construction and Dual-luciferase reporter assay. The wild-type vector of STAT-3 3'-UTR (named as STAT-3 3'-UTR WT) was amplified from cDNA using the primers: STAT3, forward, 5'-CCGCTCGAGTGATTCTGGCTTCCT TCCTG-3' (XhoI) and reverse, 5'-ATAAGAATGCGGCCGC CGAGGGCAGACTCAAGTTTATC-3' (NotI). The mutated vector of STAT-3 3'-UTR was acquired using the primers: STAT3-forward-MUT: 5'-AGGTCCCTCATCCATACACA GATAGGAATCCTGGTCTC-3' (mutation site), STAT3reverse-MUT: 5'-GAGACCAGGATTCCTATCTGTGTATG GATGAGGGACCT-3' (mutation site). Then the sequences were cloned into psiCHECK-2 vector. All the inserted sequences were verified by DNA sequencing (Sangon Biotech, Co.,Ltd., Shanghai, China). The logarithmic phase of HEK293T cells were seeded in a 96-well plate $24 \mathrm{~h}$ before transfection, STAT-3 3'-UTR vector (WT or MUT) and miR-495 mimics or negative control (NC) were co-transfected into HEK293T cells using Lipofectamine 2000. After transfection for $24 \mathrm{~h}$, cells were lysed mildly and collected and then luciferase (firefly and Renilla) activities were measured by fluorospectrophotometer.

5-AzaC treatment. Cells/well BC cells $\left(5 \times 10^{5}\right)(\mathrm{MCF}-7$ and HCC1973) were seeded in 6-well plates and cultured for $12 \mathrm{~h}$. Then, MCF-7 and HCC1973 cells were treated with $5 \mu \mathrm{M} 5$-AzaC (Sigma-Aldrich, St. Louis, MO, USA) for $72 \mathrm{~h}$. Treated cells were prepared for sodium bisulfite sequencing method (BSP), western blot analysis, RT-qPCR and detected the cell motility.

Sodium bisulfite sequencing method (BSP). Human genomic DNA incubated with SssI methyltransferase (Thermo Fisher Scientific, Waltham, MA USA) was used as positive control. 
The genomic DNA was isolated using Biospin ${ }^{\mathrm{TM}}$ cell Genomic DNA isolation kit (Hangzhou Bioer Technology, Co., Ltd., Hangzhou, China), and then treated with EpiTect Bisulfite kit (Qiagen, Hilden, Germany) and collected the genomic DNA treated with bisulfate for DNA sequencing. miR-495 promoter fragments were amplified using Takara Taq $^{\mathrm{TM}}$ Hot Start Version (Takara Bio, Dalian, China) and PCR cyclic condition: initial incubation at $95^{\circ} \mathrm{C}$ for $5 \mathrm{~min}$; followed by 40 cycles of denaturation, at $95^{\circ} \mathrm{C}$ for $30 \mathrm{sec}$, annealing, $56^{\circ} \mathrm{C}$ for $30 \mathrm{sec}$ and extension, $72^{\circ} \mathrm{C}$ for $30 \mathrm{sec}$; and finally extension, $72^{\circ} \mathrm{C}$ for $10 \mathrm{~min}$. The miR-495 promoter primer sequences were designed by software oligo 7.01, and the primer sequences are: miR-495 promoter forward, 5'-TGTAGAGGGGGTAGATTTGTTAGAT-3' and reverse, 5'-CTAAAATTACACCCAAACCAAAAC-3'.

Transwell assays. The cells treated with 5-AzaC or transfected with miR-495 mimic or siSTAT-3 in RPMI-1640 were seeded in upper chamber of $80 \mu \mathrm{l}$ coated Matrigel matrix (Corning Costar Corp., Corning, NY, USA) for invasion assay or noncoated for migration assay and $0.6 \mathrm{ml}$ of complete medium was added to the lower chamber. Non-treated cells or transfected with scrambled RNA was used as a negative control. After cultured for $24 \mathrm{~h}$, cells were fixed by $4 \%$ paraformaldehyde. Cells were dyed with $0.1 \%$ crystal violet (Beyotime Institute of Biotechnology) at room temperature for $30 \mathrm{~min}$ and redundant crystal violet was washed with $75 \%$ alcohol for 30 sec. Cells located on upper surface of the chamber were removed, and invasive/migratory cells located on the lower surface of the chamber remained. For quantification, the migratory cells and invasive cells were counted under x100 microscope in four random fields per well. Each experiment was repeated three times.

EdU assay. The cell proliferation rates were detected following the protocol of the Cell Light ${ }^{\mathrm{TM}}$ EdU Apollo ${ }^{\circledR} 488$ In Vitro Imaging kit (cat. no. C00051; Guangzhou RiboBio, Co., Ltd., Guangzhou, China). The cells were treated with 5-AzaC or transfected with miR495 mimic, siSTAT-3 or NC for $48 \mathrm{~h}$ in 6-well plates, and then $50 \mu \mathrm{M}$ of EdU was added into the medium. After incubation for $2 \mathrm{~h}$, cells were fixed with $4 \%$ paraformaldehyde $(50 \mu \mathrm{l} /$ well) for $20 \mathrm{~min}$ and then incubated with glycine $(2 \mathrm{mg} / \mathrm{ml})$ for $5 \mathrm{~min}$. The cells were washed with PBS twice and stained with $200 \mu \mathrm{l} 1 \mathrm{X}$ Apollo solution at $37^{\circ} \mathrm{C}$ for $30 \mathrm{~min}$ in the dark. After washing with $0.5 \%$ Triton X-100 in PBS for 5 min, cells were analyzed by FACScan instrument (Becton-Dickinson, San Diego, CA, USA) and data were analyzed using CellQuest software.

Flow cytometry assay. Cell apoptosis was evaluated with Annexin V-FITC/PI kit (Sigma-Aldrich) and flow cytometry assay according to the manufacturer's instructions. Briefly, cells were seeded in 6 -well plates $\left(1 \times 10^{4}\right.$ cells/well $)$ after being treated with 5 -AzaC or transfected with miR-495 mimic, siSTAT-3 or NC. The cells were then washed with ice-cold PBS and were resuspended in binding buffer prior to addition with Annexin V-FITC solution and PI. After the incubation for $15 \mathrm{~min}$ in the dark, the apoptotic rate was determined using the flow cytometer (BD Biosciences, San Jose, CA, USA).
Hoechst staining assay. The cells were treated with 5-AzaC or transfected with miR495 mimic, siSTAT-3 or NC for $48 \mathrm{~h}$ in 6-well plates. The medium was removed and the cells were washed with PBS. Hoechst $33342(1 \mu \mathrm{M})$ was added into PBS for $10 \mathrm{~min}$ at $37^{\circ} \mathrm{C}$. The changes in nuclear morphology were analyzed by fluorescence microscopy.

Bioinformatic and statistical analysis. Online miRNA databases (miRBase, TargetScan, miRanda and PicTarget) were used for the prediction of miR-495 target genes and possible miRNAs regulating STAT-3. All data were analyzed by using Graphpad Prism 5.01 software (GraphPad Software, Inc., La Jolla, CA, USA). The unpaired Student's t-test (two-tailed) was used to analyze the differences the two groups. All data are expressed as the mean $\pm \mathrm{SD}$. $\mathrm{P}<0.05, \mathrm{P}<0.01, \mathrm{P}<0.001$.

\section{Results}

miR-495 is downregulated and STAT-3 is upregulated in the $B C$ tissue and cell lines. To explore the conceivable relationship between miR-495 and STAT-3 in BC, the expression of miR-495 and STAT-3 in BC patient tumor and adjacent tissue was measured by RT-qPCR. The data showed that the expression of miR-495 was significantly downregulated in BC patients tissue compared with adjacent tissue $(\mathrm{P}<0.001$; Fig. 1A), while STAT-3 presented overexpressed in BC tissue $(\mathrm{P}<0.01$; Fig. 1B). Similar results were also observed in BC cell lines (MCF-7 and HCC1973) in comparison with the normal mammary epithelial cells (MCF10A) ( $\mathrm{P}<0.001$; Fig. $1 \mathrm{C}$ and D). Furthermore, we determined the protein level of STAT-3 in BC tissues and the cell lines using western blotting. As shown in Fig. 1E, the expression of STAT-3 was obviously upregulated in representative tumor tissues compared with the adjacent normal tissues. Consistently, the expression of STAT-3 presented higher expression in BC cell lines than that in normal epithelial cells (Fig. 1F). Preliminary findings suggest that miR-495 might inhibit the STAT-3 expression in $\mathrm{BC}$ tissue and cell lines.

miR-495 directly targeted the 3'-UTR of STAT-3. As known, miRNAs exert their function by affecting their target gene expression. To illuminate the regulation mechanism of STAT-3 expression by miR-495 at the post-transcriptional level, miR-495 mimic and its negative control (NC) were transfected into MCF-7 and HCC-1973 cells, respectively. The expression of STAT-3 (both mRNA and protein level) was significantly lower in miR-495 mimic transfected cells than NC group and parental cells (Fig. 2A-C). To find the possible target genes of miR-495 and possible miRNAs inhibiting STAT-3 expression, we predicted it using bioinformatics analysis (Fig. 2D). To verify the prediction, a luciferase reporter assay was performed in HEK293T cells. The fluorescence intensity was significantly decreased after con-transfection with psiCHECK-2/STAT-3 3'-UTR-WT reporter plasmid and miR-495 mimic in comparison with control cells (Fig. 2E). Our results demonstrated that miR-495 specifically binds to the 3'-UTR of STAT-3 mRNA degrading it.

Silencing of miR-495 is mediated by hypermethylation. To demonstrate the interaction between the expression of 
A

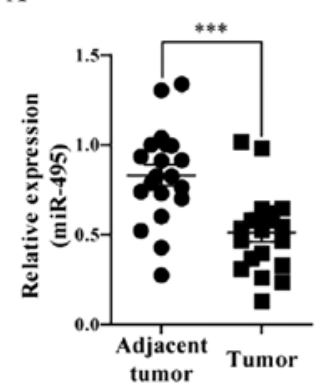

B

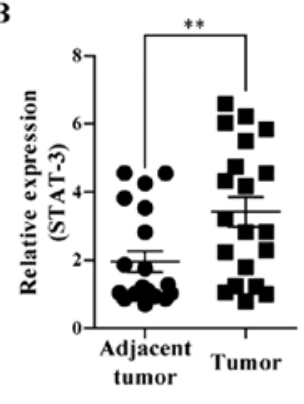

C

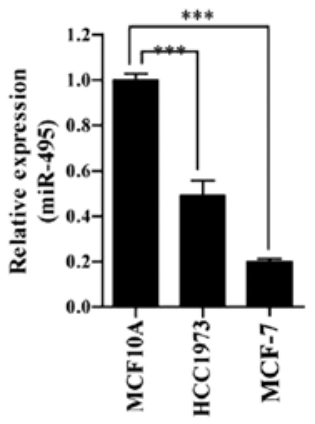

D

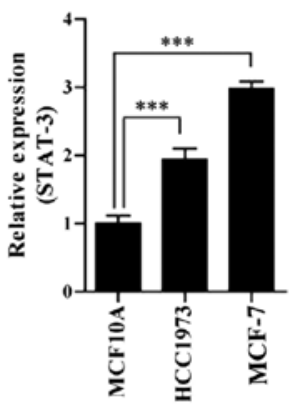

E
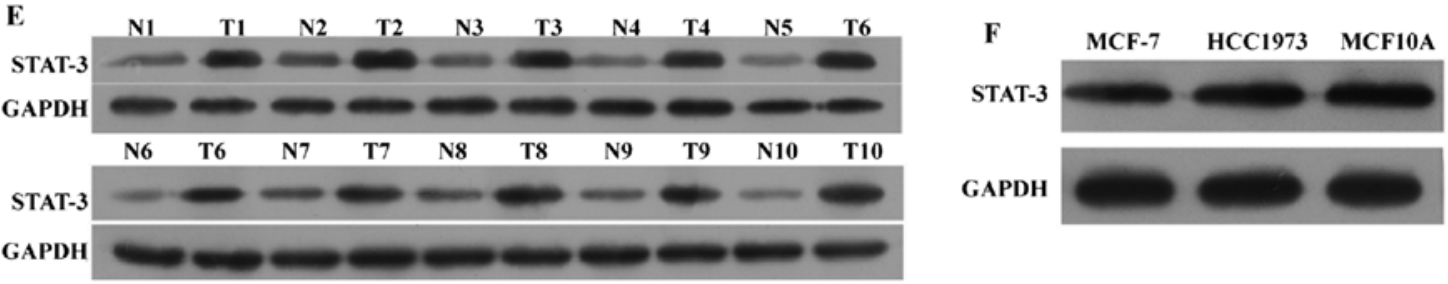

Figure 1. miR-495 was downregulated and STAT-3 was upregulated in the breast cancer tissue and cells. (A) The mRNA levels of miR-495 and (B) STAT-3 in breast cancer patients tissue compared with adjacent tissue were analyzed by RT-qPCR. ${ }^{* *} \mathrm{P}<0.01$. (C) The mRNA levels of miR-495 and (D) STAT-3 in the immortalized breast epithelial (MCF10A) and human breast cancer cells lines (MCF-7 and HCC1973) were analyzed by RT-qPCR. The protein level of STAT-3 in (E) breast cancer patients tissue and (F) human breast cancer cells lines (MCF-7 and HCC1973) were analyzed by western blotting. All data are presented as mean value $\pm \mathrm{SD}$ from 3 independent experiments. ${ }^{* * *} \mathrm{P}<0.001$. GAPDH was used as an internal control.
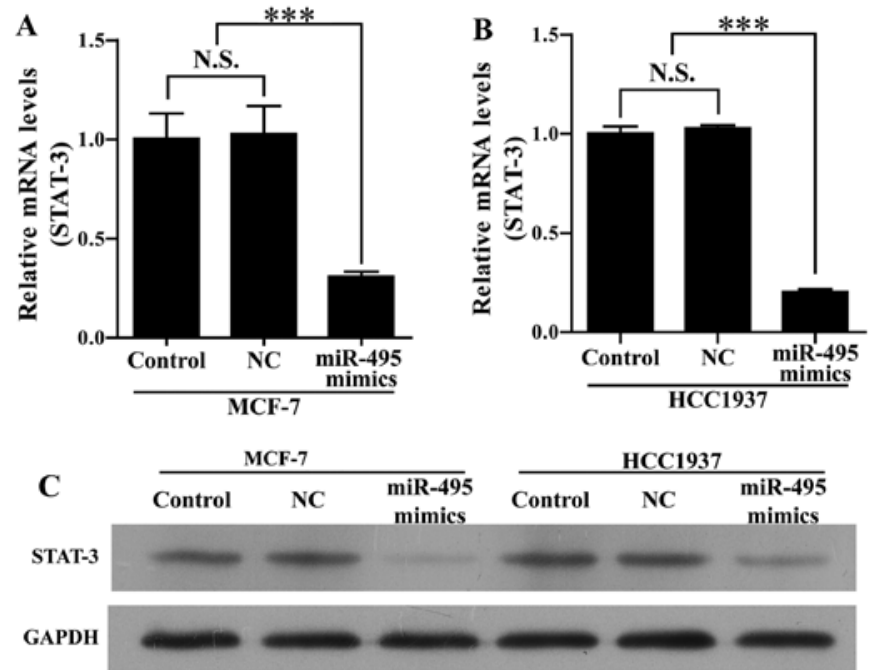

\section{3'-UUCUUCACGUGGUACAAACAAA-5' miR-495<smiles>C=CC(=C)C(=C)C(=C)C(=C)C(=C)C(=C)C(=C)C(=C)C(=C)C(=C)C(=C)C(=C)C</smiles> \\ 5'-AGGUCCCUCAUCCUGUUUGUUU-3' STAT3 WT \\ 5'-AGGUCCCUAAAGAAACACCGAG-3' STAT3 MUT}

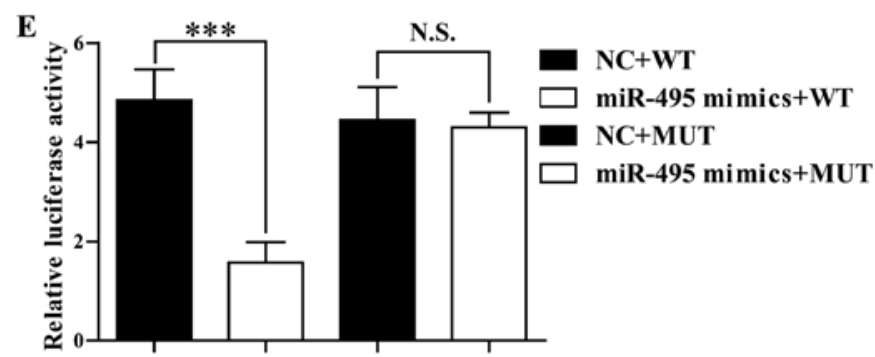

Figure 2. miR-495 directly targets the 3'-UTR of STAT-3. (A) The mRNA levels of STAT-3 in breast cancer cell lines MCF-7 transfected with miR-495 mimic or NC were analyzed by RT-qPCR. (B) The mRNA levels of STAT-3 in breast cancer cell lines HCC1973 transfected with miR- 495 mimic or NC were analyzed by RT-qPCR. (C) The protein levels of STAT-3 in breast cancer cell lines MCF-7 and HCC1973 transfected with miR-495 mimic or NC were analyzed by western blot analysis. (D) The binding site of miR-495 and the prediction target genes (STAT-3) through TargetScan web and the sequence of wild-type and mutation STAT-3 3'-UTR. (E) The wild-type (STAT-3 3'-UTR-WT) or mutant (STAT-3 3'-UTR-MUT) reporter plasmids and miR-495 mimic or NC were co-transfected into $293 \mathrm{~T}$ cells. The normalized luciferase activity in the control group was set as relative luciferase activity. All data are presented as mean value $\pm \mathrm{SD}$ from 3 independent experiments. ${ }^{* * *} \mathrm{P}<0.001$. 
A

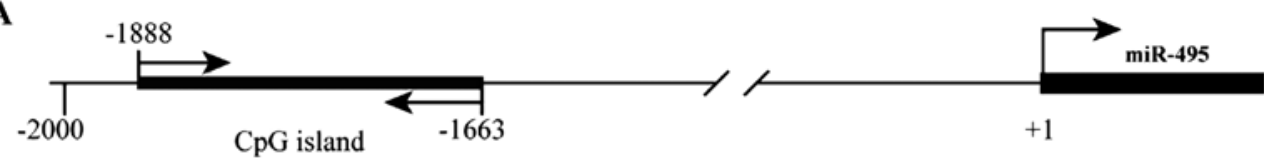

B CpG island

$-1663$

5-Aza

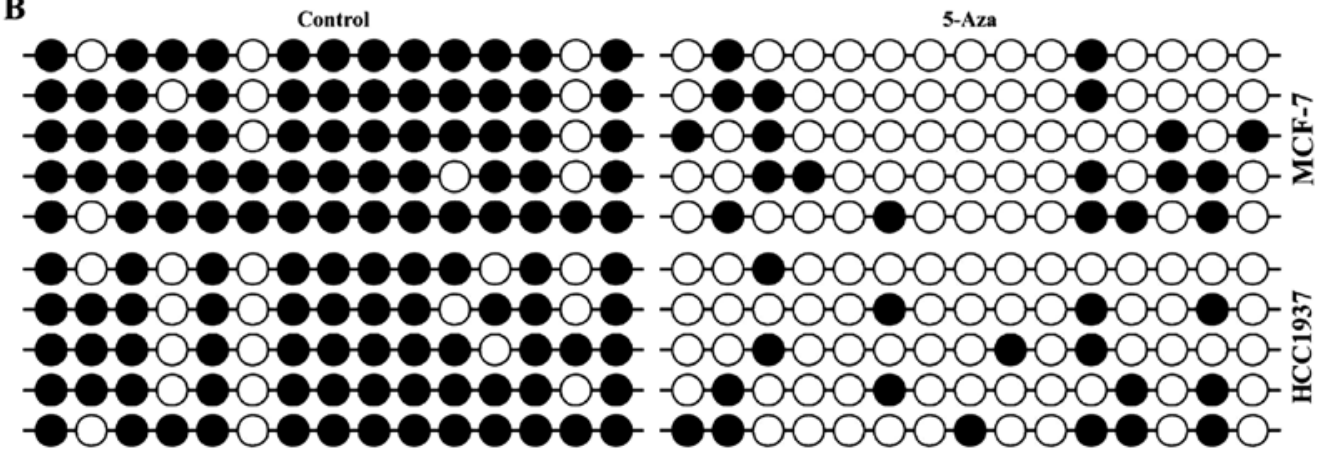

C

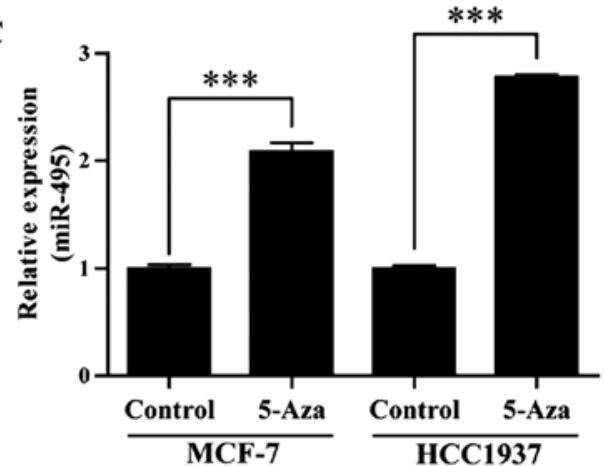

D

$\mathbf{E}$

MCF-7
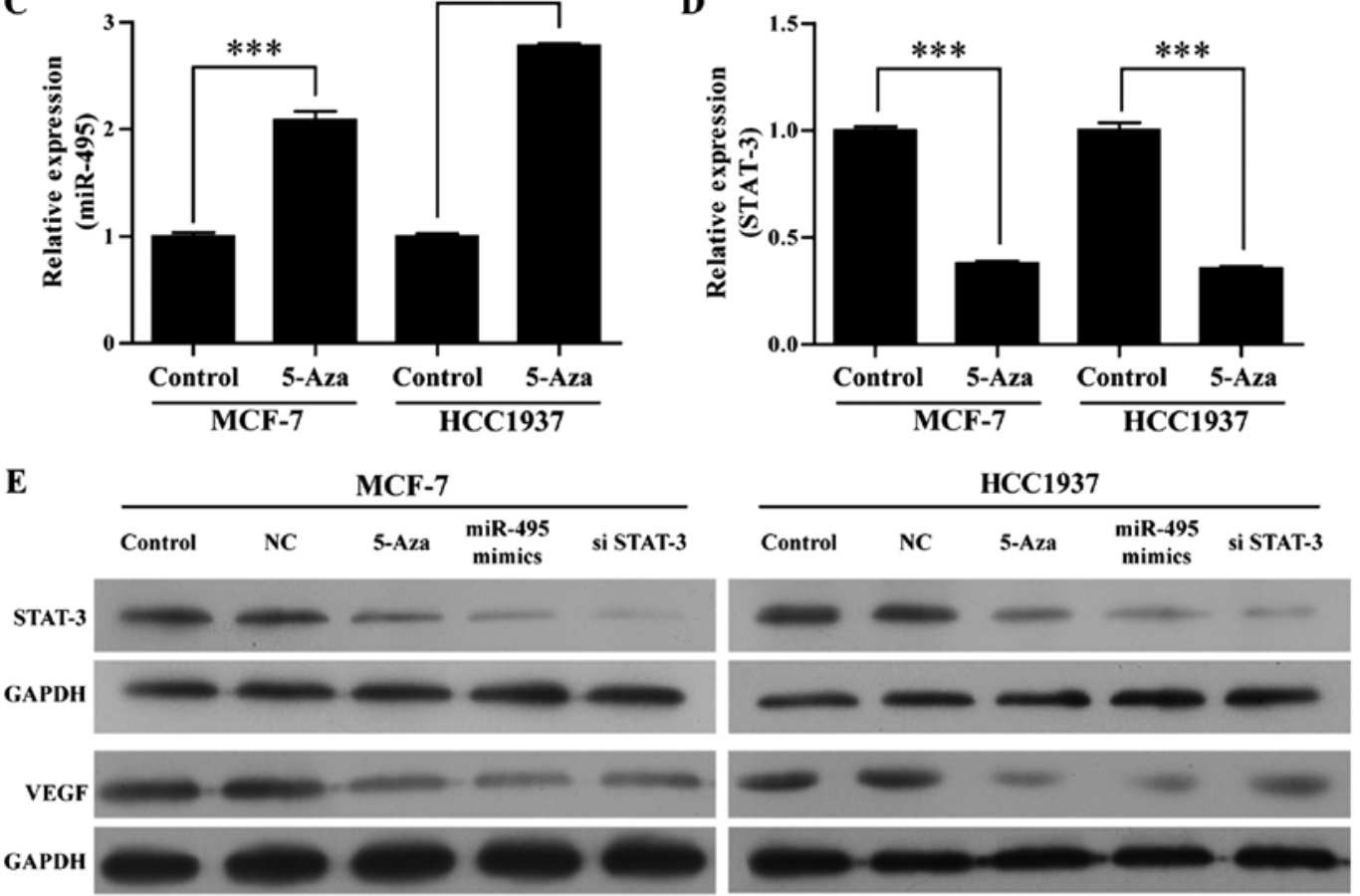

Figure 3. 5-AzaC reverses the methylation of the miR-495 gene promoter and re-expression of miR-495 expression but downregulates STAT-3 and its downstream target VEGF. (A) The section of methylation of miR-495 gene promoter. (B) BSP analysis of miR-495 in breast cancer cell lines (MCF-7 and HCC1973), with or without 5-AzaC treatment. Open and filled circles represent unmethylated and methylated $\mathrm{CpG}$ sites, respectively. The black line between circles represent the base pairs between each CpG sites, each horizontal row represents a single clone. There are $15 \mathrm{CpG}$ sites. (C) The mRNA levels of miR-495 in breast cancer cell lines MCF-7 and HCC1973 with or without 5-AzaC treatment were analyzed by RT-qPCR. (D) The mRNA levels of STAT-3 in breast cancer cell lines MCF-7 and HCC1973 with or without 5-AzaC treatment were analyzed by RT-qPCR. (E) The protein levels of STAT-3 and VEGF after treated with 5-AzaC or transfect with miR-495 mimic or siSTAT-3 in breast cancer cells (MCF-7 and HCC1973) were analyzed by western blot analysis. All quantitative data are presented as mean value $\pm \mathrm{SD}$ from 3 independent experiments. ${ }^{* * *} \mathrm{P}<0.001$ vs. control.

miR-495 and miR-495 promoter methylation, we predicted the section of methylation using CpGProD (http://doua.prabi. fr/software/cpgprod_query). As shown in Fig. 3A, -2000 to -1663 bp may be the section of methylation of miR-495. Next we also detected the methylation state of the miR-495 gene CpG islands by BSP. As shown in Fig. 3B, after incubation with 5-AzaC, the methylation state of the miR-495 geneCpG islands was markedly reduced compared with parental cells. As known, 5-AzaC is the most commonly DNA methyltransferase inhibitor. Therefore, just as the result shown, 5-AzaC could demethylate the miR-495 gene $\mathrm{CpG}$ islands and re-express miR-495 and then inhibit STAT-3 again in BC cells.
To further evaluate whether demethylation of miR-495 gene could affect the levels of STAT-3, we treated the BC cells lines with 5-AzaC. The level of miR-495 in BC cells was markedly re-expressed via 5-AzaC (Fig. 3C; $\mathrm{P}<0.01$ ). Furthermore, in order to evaluate whether re-expression of miR-495 could inhibit STAT-3 by treated with 5-AzaC in BC cells, we detected the expression levels of STAT-3 after being treated with 5-AzaC. As shown in Fig. 3D, the mRNA and protein level of STAT-3 was markedly reduced compared with parental cells $(\mathrm{P}<0.001)$. In western blot assay, BC cells treated with 5-AzaC obviously downergulated the expression of STAT-3 and its downstream target VEGF (Fig. 3E). Similar 


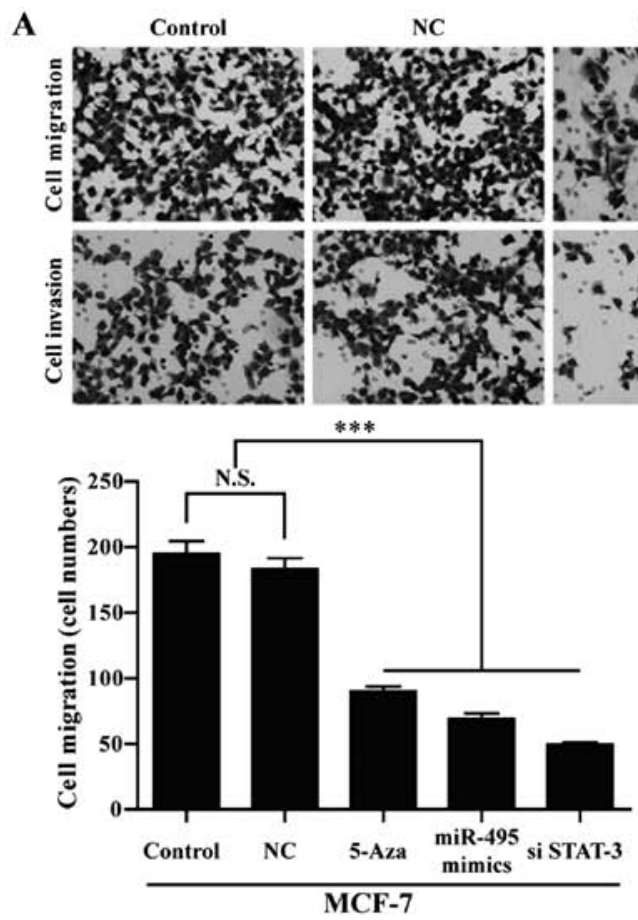
5-Aza miR-495 mimics si STAT-3
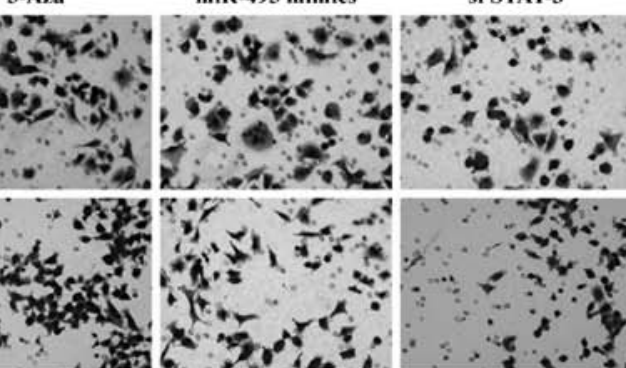

. 1.6:-

紊

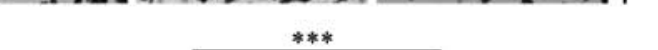

B
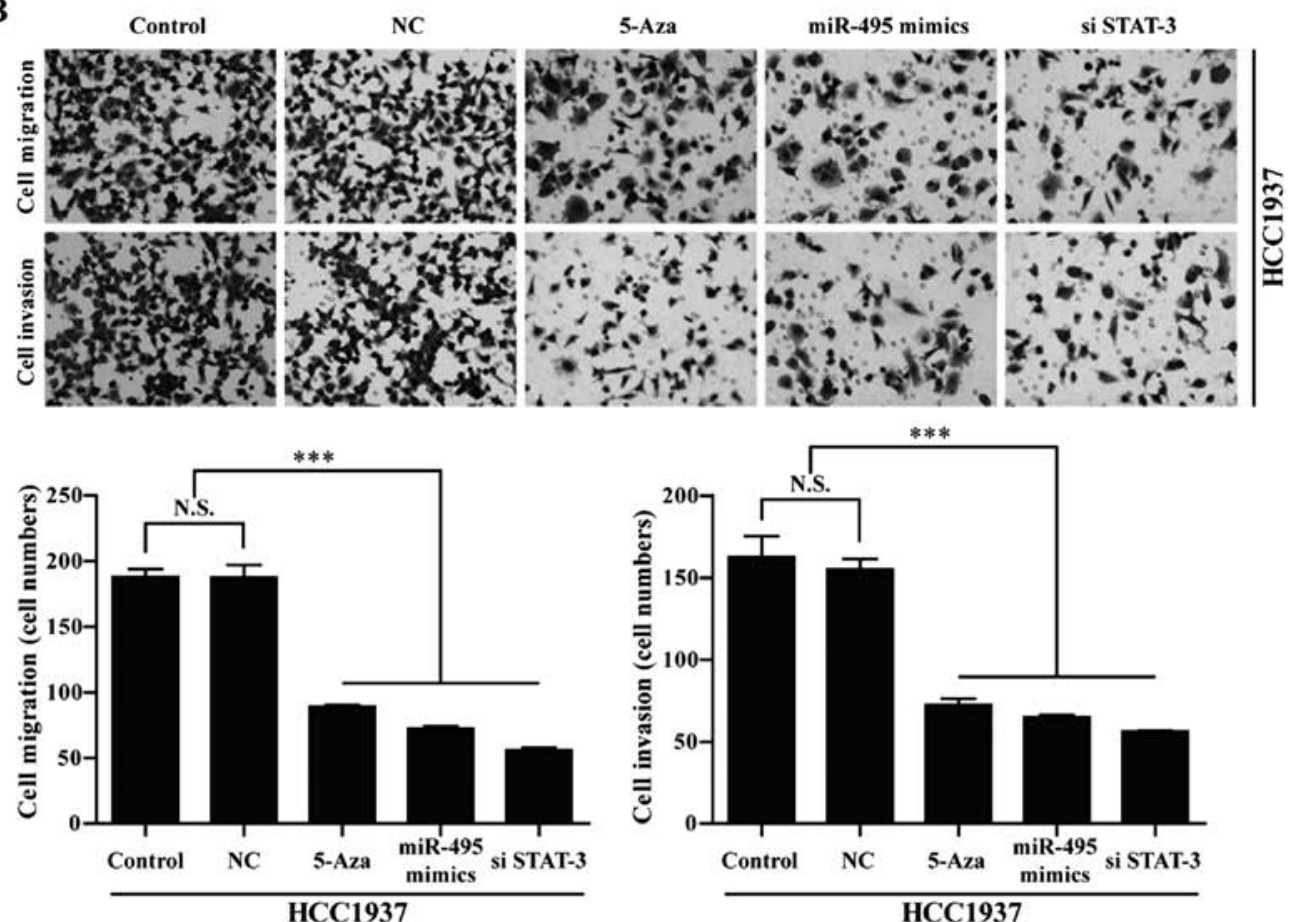

Figure 4. Treatment with 5-AzaC suppresses breast cancer invasion and metastasis. After treated with 5-AzaC or transfected with miR-495 mimic and siSTAT-3 the breast cancer cells MCF-7 (A) and HCC1973 (B) were subjected to Transwell assays. Representative photographs of migrating and invading cells. Average cell numbers for migrating and invading cells (mean value \pm SD) from three independent experiments are shown above. All quantitative data are presented as mean value $\pm \mathrm{SD}$ from 3 independent experiments. ${ }^{* * *} \mathrm{P}<0.001$ vs. control or NC.

results were also found in miR-495 mimic and siSTAT-3 groups. Our results demonstrated that hypermethylationmediated silencing of miR-495 lead to the re-expression of oncogene STAT-3, and 5-AzaC could significantly reverse the expression of miR-495 and STAT-3.

Treatment with 5-AzaC, miR-495 mimics or siSTAT-3 suppress $B C$ migration and invasion. To investigate how miR-495 affects biological functions of BC, we first analyzed

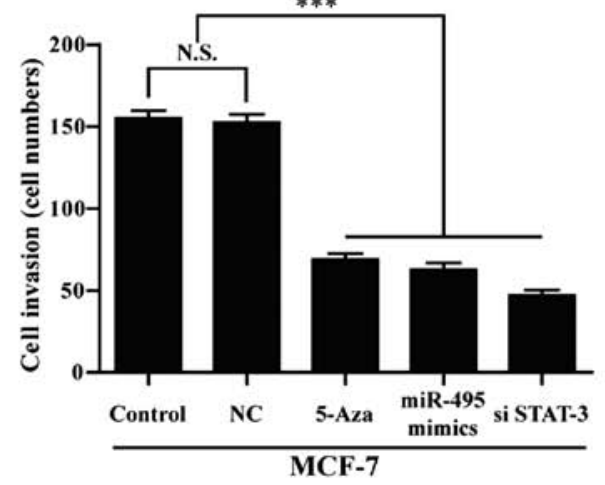

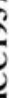


A
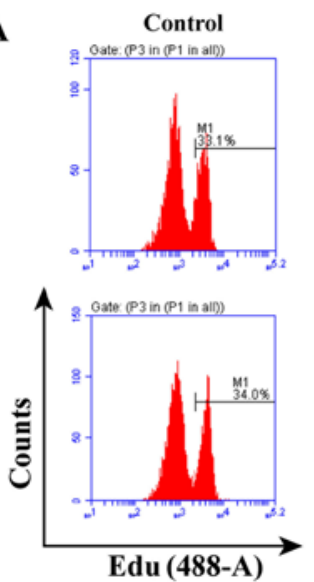

B

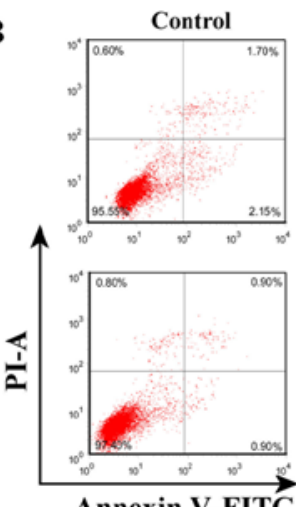

C

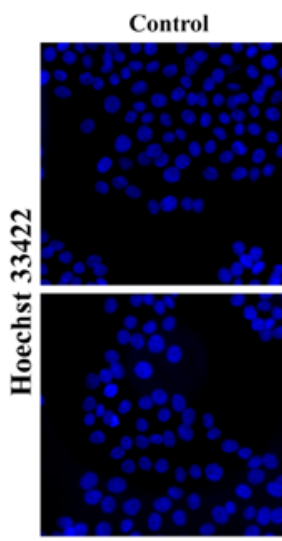

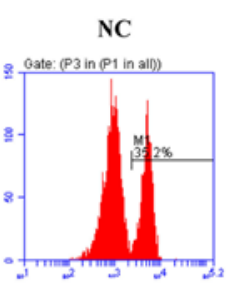
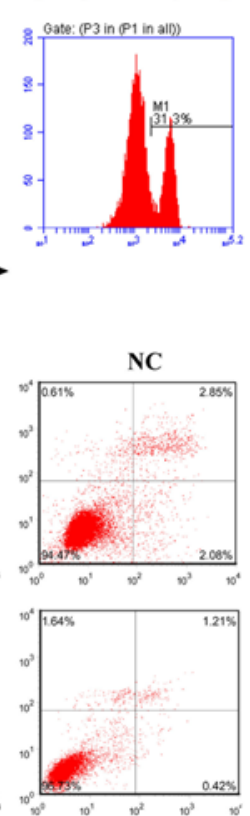

\section{.}

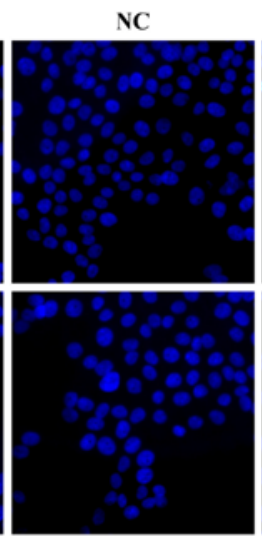

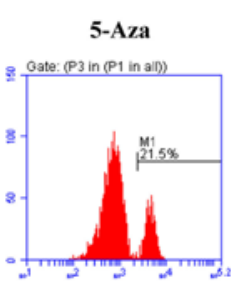
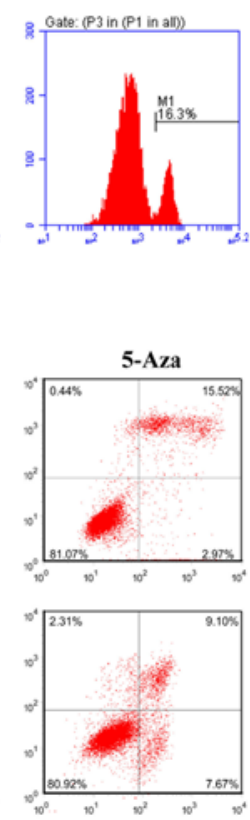

5-Aza

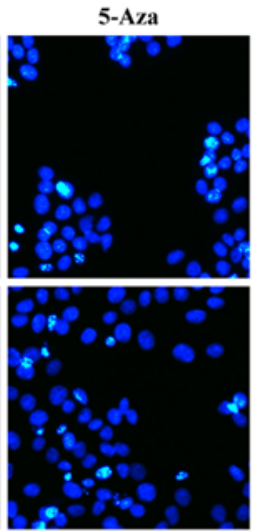

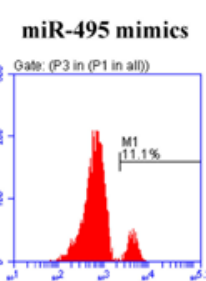
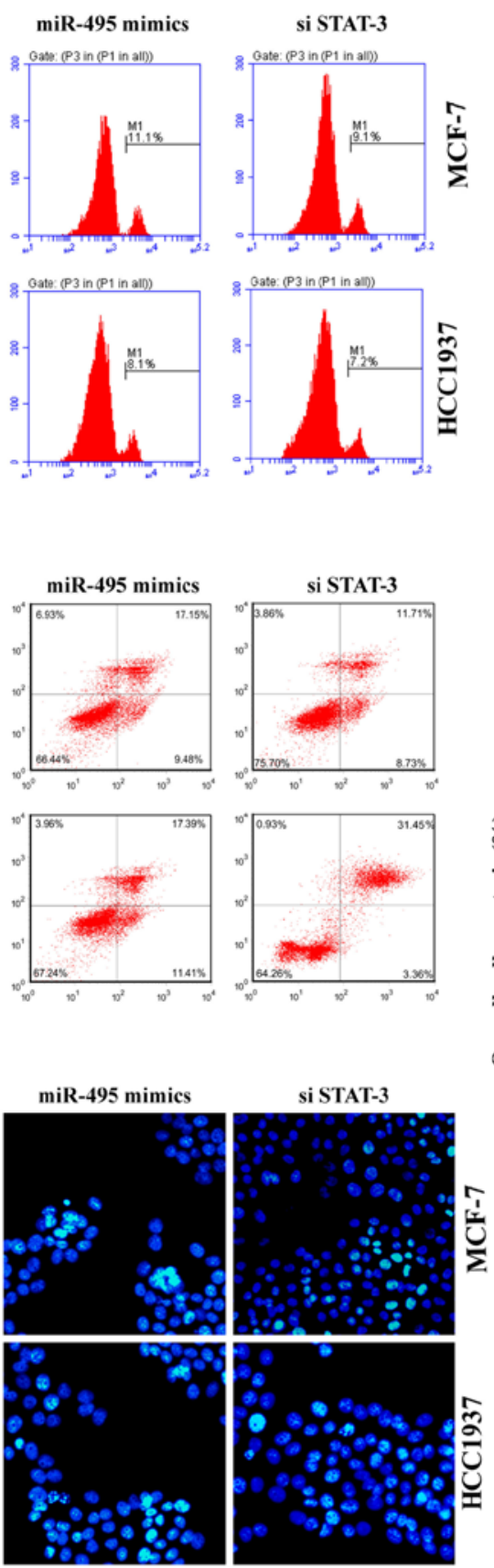
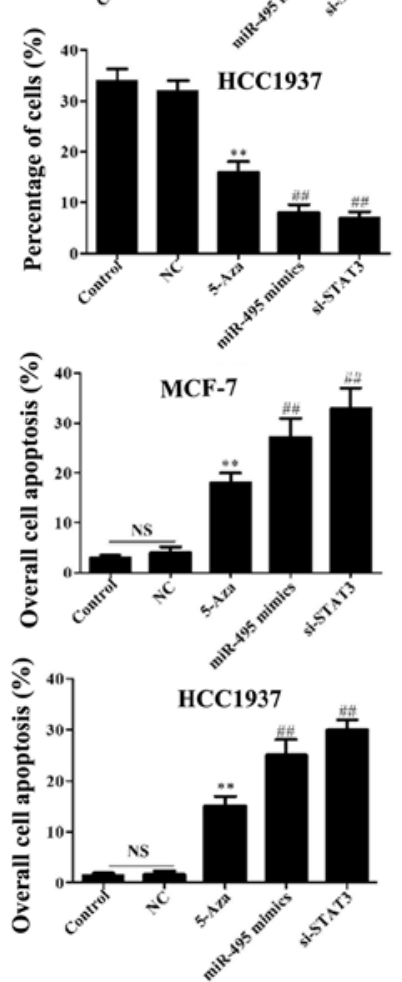

Figure 5. Treatment with 5-AzaC, miR-495 or siSTAT-3 inhibits cell proliferation and promotes breast cancer cell apoptosis in breast cancer cells. (A) After treated with 5-AzaC or transfect with miR-495 mimic and siSTAT-3 breast cancer cells were subjected to Edu labeling. Representative data of FACS analyses of cell proliferation in MCF-7 and HCC1973 cells. After treated with 5-AzaC or transfect with miR-495 mimic and siSTAT-3 breast cancer cells were subjected to flow cytometry (B) and Hoechst staining (C).

ated the cell proliferation rates using EdU flow cytometry assay in MCF-7 and HCC1937 cells. As shown in Fig. 5A, the percentage of positive fluorescence was obviously decreased in $\mathrm{BC}$ cells treated with 5-AzaC $(\mathrm{P}<0.01)$, miR-495 mimics $(\mathrm{P}<0.01)$ or siSTAT-3 $(\mathrm{P}<0.01)$ compared with that of the control groups. As shown in Fig. 5B, more cells represented apoptotic status, including early apoptosis (Annexin $\mathrm{V}^{+} / \mathrm{PI}^{-}$) and late apoptosis (Annexin $\mathrm{V}^{+} / \mathrm{PI}^{+}$) in $\mathrm{BC}$ cells after treated with 5-AzaC $(\mathrm{P}<0.01)$, miR-495 mimics $(\mathrm{P}<0.01)$ or siSTAT-3 $(\mathrm{P}<0.01)$ compared with controls. In Hoechst staining assay, BC cells treated with 5-AzaC, miR-495 mimics or siSTAT-3 presented a significantly increased cell apoptosis than the untreated cells or negative control cells (Fig. 5C).

\section{Discussion}

DNA methylation is known to be a part of the epigenetic gene regulatory machinery. $\mathrm{CpG}$ islands are found in $\sim 60 \%$ of the human gene promoter regions, including both protein coding and non-protein-coding genes such as miRNA genes $(27,28)$. Recent studies have highlighted the potential role of epigenetic silencing of miRNA genes in tumor drug resistance, tumori- 
genesis and tumor progression and as a diagnostic biomarker for cancer (29-32). For example, numerous tumor suppressor genes are methylated and thus, transcriptionally silenced in chronic lymphocytic leukemia (33). Among these, miR-495 stands out as one of most frequently identified miRNAs with methylation in their promoter regions in gastric cancer cells (24). Consistent with these observations, downregulation of the precursors of miR-495 in metastatic prostate cancer cells have been documented by several laboratories (34). In various human cancers, singular activation of STAT- 3 pathway sufficiently induced cell tumorigenesis both in vitro and in vivo (35). The constitutively active STAT-3 pathway may be involved in oncogenesis by enhancing cell proliferation, promoting angiogenesis, invasion, metastasis and acquired multidrug resistance (36).

Our results indicated that the hypermethylation of the miR-495 gene CpG islands leads to the silencing of miR-495 and upregulation of STAT-3. The DNA methyltransferase inhibitor 5-AzaC is the most commonly used demethylating drug able to reverse DNA methylation resulting in gene re-expression. We speculated that it has potential as a target for combine therapy in human cancer. Many studies have confirmed that the tumor suppressor gene expression could re-expressed by 5-AzaC (37-39). Our findings showed that use of 5-Aza-dC robustly increased miR-495 levels by demethylation of DNA and then strongly inhibited STAT-3 expression and cell motility in BC. In addition, VEGF, as a downstream target of STAT-3 (40), was also downregulated in BC cells after treated with 5-AzaC, miR-495 mimics or siSTAT-3, respectively.

Overall, these observations suggest that one of the mechanisms by which miR-495 overexpression induced demethylation agent 5-AzaC inhibited $\mathrm{BC}$ cell proliferation, invasion, and promoted apoptosis is due to the inhibition of STAT-3 and associated with a decrease in VEGF expression. However, it should be emphasized that the present study merely provides a piece of evidence for the effect of methylation of miR-495 in regulating tumor progression of $\mathrm{BC}$, and we should not generalize this conclusion solely based on the results from several cell lines. To confirm this issue with sufficient data, rigorous experimental studies in more cells or models are still required.

In conclusion, taken together, the present study provides strong evidence that methylation-silencing of miR-495 is an important epigenetic mechanism favoring cancer cell growth and survival in BC and likely in other cancers as well, and demethylation to reactivate expression of miR-495 genes may be a new combine therapy for human BC.

\section{References}

1. Torre LA, Bray F, Siegel RL, Ferlay J, Lortet-Tieulent J and Jemal A: Global cancer statistics, 2012. CA Cancer J Clin 65 87-108, 2015.

2. Agarwal G, Pradeep PV, Aggarwal V, Yip CH and Cheung PS: Spectrum of breast cancer in Asian women. World J Surg 31: 1031-1040, 2007

3. O'Driscoll L and Clynes M: Biomarkers and multiple drug resistance in breast cancer. Curr Cancer Drug Targets 6: 365-384, 2006.

4. Li B, Lu Q, Song ZG, Yang L, Jin H, Li ZG, Zhao TJ, Bai YF, Zhu J, Chen HZ, et al: Functional analysis of DNA methylation in lung cancer. Eur Rev Med Pharmacol Sci 17: 1191-1197, 2013.
5. Arrigoni E, Galimberti S, Petrini M, Danesi R and Di Paolo A: ATP-binding cassette transmembrane transporters and their epigenetic control in cancer: An overview. Expert Opin Drug Metab Toxicol 12: 1419-1432, 2016.

6. Yen CY, Huang HW, Shu CW, Hou MF, Yuan SS, Wang HR, Chang YT, Farooqi AA, Tang JY and Chang HW: DNA methylation, histone acetylation and methylation of epigenetic modifications as a therapeutic approach for cancers. Cancer Lett 373: 185-192, 2016.

7. Kozaki K and Inazawa J: Tumor-suppressive microRNA silenced by tumor-specific DNA hypermethylation in cancer cells. Cancer Sci 103: 837-845, 2012.

8. Liu SL, Sui YF and Lin MZ: MiR-375 is epigenetically downregulated due to promoter methylation and modulates multi-drug resistance in breast cancer cells via targeting YBX1. Eur Rev Med Pharmacol Sci 20: 3223-3229, 2016.

9. Ye XM, Zhu HY, Bai WD, Wang T, Wang L, Chen Y, Yang AG and Jia LT: Epigenetic silencing of miR-375 induces trastuzumab resistance in HER2-positive breast cancer by targeting IGF1R. BMC Cancer 14: 134, 2014.

10. Li X, Wu Y, Liu A and Tang X: MiR-27b is epigenetically downregulated in tamoxifen resistant breast cancer cells due to promoter methylation and regulates tamoxifen sensitivity by targeting HMGB3. Biochem Biophys Res Commun 477: 768-773, 2016.

11. He DX, Gu XT, Li YR, Jiang L, Jin J and Ma X: Methylationregulated miR-149 modulates chemoresistance by targeting GlcNAc N-deacetylase/N-sulfotransferase-1 in human breast cancer. FEBS J 281: 4718-4730, 2014.

12. Hu H, Li S, Cui X, Lv X, Jiao Y, Yu F, Yao H, Song E, Chen Y, Wang M, et al: The overexpression of hypomethylated miR-663 induces chemotherapy resistance in human breast cancer cells by targeting heparin sulfate proteoglycan 2 (HSPG2). J Biol Chem 288: 10973-10985, 2013.

13. He DX, Gu XT, Jiang L, Jin J and Ma X: A methylation-based regulatory network for microRNA $320 \mathrm{a}$ in chemoresistant breast cancer. Mol Pharmacol 86: 536-547, 2014.

14. Xu Q, Jiang Y, Yin Y, Li Q, He J, Jing Y, Qi YT, Xu Q, Li W, $\mathrm{Lu} \mathrm{B}$, et al: A regulatory circuit of miR-148a/152 and DNMT1 in modulating cell transformation and tumor angiogenesis through IGF-IR and IRS1. J Mol Cell Biol 5: 3-13, 2013.

15. Li JZ, Wang ZL, Xu WH, Li Q, Gao L and Wang ZM: MicroRNA495 regulates migration and invasion in prostate cancer cells via targeting Akt and mTOR signaling. Cancer Invest 34: 181-188, 2016.

16. Lee SH, Jung YD, Choi YS and Lee YM: Targeting of RUNX3 by miR-130a and miR-495 cooperatively increases cell proliferation and tumor angiogenesis in gastric cancer cells. Oncotarget 6: 33269-33278, 2015.

17. Xu YY, Tian J, Hao Q and Yin LR: MicroRNA-495 downregulates FOXC1 expression to suppress cell growth and migration in endometrial cancer. Tumour Biol 37: 239-251, 2016.

18. Cao M, Nie W, Li J, Zhang Y, Yan X, Guan X, Chen X, Zen K, Zhang CY, Jiang X, et al: MicroRNA-495 induces breast cancer cell migration by targeting JAM-A. Protein Cell 5: 862-872, 2014.

19. Mao Y, Li L, Liu J, Wang L and Zhou Y: MiR-495 inhibits esophageal squamous cell carcinoma progression by targeting Akt1. Oncotarget 7: 51223-51236, 2016.

20. Lv C, Bai Z, Liu Z, Luo P and Zhang J: MicroRNA-495 suppresses human renal cell carcinoma malignancy by targeting SATB1. Am J Transl Res 7: 1992-1999, 2015.

21. Chen SM, Chen HC, Chen SJ, Huang CY, Chen PY, Wu TW, Feng LY, Tsai HC, Lui TN, Hsueh C, et al: MicroRNA-495 inhibits proliferation of glioblastoma multiforme cells by downregulating cyclin-dependent kinase 6. World J Surg Oncol 11: 87, 2013.

22. Jiang X, Huang H, Li Z, He C, Li Y, Chen P, Gurbuxani S, Arnovitz S, Hong GM, Price C, et al: MiR-495 is a tumorsuppressor microRNA down-regulated in MLL-rearranged leukemia. Proc Natl Acad Sci USA 109: 19397-19402, 2012.

23. Chu H, Chen X, Wang H, Du Y, Wang Y, Zang W, Li P, Li J, Chang J, Zhao G and Zhang G: MiR-495 regulates proliferation and migration in NSCLC by targeting MTA3. Tumour Biol 35: 3487-3494, 2014

24. Li Z, Zhang G, Li D, Jie Z, Chen H, Xiong J, Liu Y, Cao Y, Jiang M, Le Z, et al: Methylation-associated silencing of miR-495 inhibit the migration and invasion of human gastric cancer cells by directly targeting PRL-3. Biochem Biophys Res Commun 456: 344-350, 2015. 
25. Xu Y, Ohms SJ,LiZ, Wang Q, Gong G, Hu Y, Mao Z, Shannon MF and Fan JY: Changes in the expression of miR-381 and miR-495 are inversely associated with the expression of the MDR1 gene and development of multi-drug resistance. PLoS One 8: e82062, 2013.

26. Song L, Li Y, Li W, Wu S and Li Z: miR-495 enhances the sensitivity of non-small cell lung cancer cells to platinum by modulation of copper-transporting P-type adenosine triphosphatase A (ATP7A). J Cell Biochem 115: 1234-1242, 2014.

27. Wang Y and Leung FC: An evaluation of new criteria for $\mathrm{CpG}$ islands in the human genome as gene markers. Bioinformatics 20: $1170-1177,2004$

28. Visone R and Croce CM: MiRNAs and cancer. Am J Pathol 174: 1131-1138, 2009.

29. Li F and Mahato RI: MicroRNAs and drug resistance in prostate cancers. Mol Pharm 11: 2539-2552, 2014

30. Jiang CG, Lv L, Liu FR, Wang ZN, Na D, Li F, Li JB, Sun Z and $\mathrm{Xu}$ HM: Connective tissue growth factor is a positive regulator of epithelial-mesenchymal transition and promotes the adhesion with gastric cancer cells in human peritoneal mesothelial cells Cytokine 61: 173-180, 2013.

31. Zang H, Wang W and Fan S: The role of microRNAs in resistance to targeted treatments of non-small cell lung cancer. Cancer Chemother Pharmacol 79: 227-231, 2016.

32. Chen X, Lu P, Wu Y, Wang DD, Zhou S, Yang SJ, Shen HY, Zhang XH, Zhao JH and Tang JH: MiRNAs-mediated cisplatin resistance in breast cancer. Tumour Biol 37: 12905-12913, 2016.

33. Wang LQ and Chim CS: DNA methylation of tumor-suppressor miRNA genes in chronic lymphocytic leukemia. Epigenomics 7: 461-473, 2015
34. Formosa A, Markert EK, Lena AM, Italiano D, Finazzi-Agro' E, Levine AJ, Bernardini S, Garabadgiu AV, Melino G and Candi E: MicroRNAs, miR-154, miR-299-5p, miR-376a, miR-376c, miR-377, miR-381, miR-487b, miR-485-3p, miR-495 and miR$654-3 \mathrm{p}$, mapped to the $14 \mathrm{q} 32.31$ locus, regulate proliferation, apoptosis, migration and invasion in metastatic prostate cancer cells. Oncogene 33: 5173-5182, 2014.

35. Buettner R, Mora LB and Jove R: Activated STAT signaling in human tumors provides novel molecular targets for therapeutic intervention. Clin Cancer Res 8: 945-954, 2002.

36. Song H, Jin $X$ and Lin J: Stat 3 upregulates MEK 5 expression in human breast cancer cells. Oncogene 23: 8301-8309, 2004.

37. Hiroki E, Suzuki F, Akahira J, Nagase S, Ito K, Sugawara J, Miki Y, Suzuki T, Sasano H and Yaegashi N: MicroRNA-34b functions as a potential tumor suppressor in endometrial serous adenocarcinoma. Int J Cancer 131: E395-E404, 2012.

38. Fan H, Zhao ZJ, Cheng YC, Shan YF, Lu ZH, Zhang JQ and Xie W: Gene induction and apoptosis in human hepatocellular carci-noma cells SMMC-7721 exposed to 5-aza-2'-deoxycytidine. Chin Med J (Engl) 120: 1626-1631, 2007.

39. Deng H, Guo Y, Song H, Xiao B, Sun W, Liu Z, Yu X, Xia T, Cui L and Guo J: MicroRNA-195 and microRNA-378 mediate tumor growth suppression by epigenetical regulation in gastric cancer. Gene 518: 351-359, 2013.

40. Bhat TA, Nambiar D, Tailor D, Pal A, Agarwal R and Singh RP. Acacetin inhibits in vitro and in vivo angiogenesis and downregulates Stat signaling and VEGF expression. Cancer Prev Res (Phila) 6: 1128-1139, 2013. 